\title{
Environmental Analysis of Toronto Neighbourhoods
}

AUTHORS: Lunjun Zhang, Jenny Baek, Evgeny Bogopolskiy, Justin Palombo

SCHOOL: Villanova College

\section{Abstract}

The increase in the industrial pollution produced by Toronto, Ontario is negatively impacting the city's environmental conditions. Although the Ministry of Environment and Climate Change has attempted to improve environment, efforts require continual re-focusing to remain effective. After research and discussion, four main factors that can affect the environment were identified: tree cover, pollutants released to air, pollutant carcinogenic Toxic Equivalency Potentials (TEP) score, and pollutant non-carcinogenic TEP score. A program which outputs a list of neighbourhoods in dire environmental condition was designed based on those four main factors and general analysis. This program uses an input of several datasets from the Open Data Toronto database. Possible solutions to pollution and areas of environmental improvement are ultimately suggested, with the objective being to raise environmental awareness.

\section{Key Words}

Environmental Analysis, Toronto Neighbourhoods, Big Data, Python

\section{Introduction}

Using general statistical analysis on relevant environmental information collected from Open Data Toronto database, the research on Toronto's environmental conditions was conducted to determine possible areas of improvement in the neighbourhoods of the city. Four main factors that could affect the environment were identified: pollutants released to air, pollutant carcinogenic TEP score, pollutant non-carcinogenic TEP score, and tree cover.

The first factor is general pollutants released to the air, which harm the air quality. The next factor is the Pollutant Carcinogenic TEP score. TEP indicate the relative health risk associated with the release of one pound of a chemical. The Pollutant Carcinogenic TEP score is a general score measuring how various chemicals affect the environment and increase the risk of cancer.

The Pollutant non-carcinogenic TEP score refers to non-carcinogenic compounds which, negatively affect the environmental conditions of the neighbourhoods. Non-carcinogenic pollutants can con- tribute to the build-up of greenhouse gases. The last factor used is the tree cover. The less tree covers a neighbourhood has, the more space there is for industries, companies, and building. Ultimately, this would result in higher production pollutants and carcinogens. The tree cover was used to resolve the problem of two neighbourhoods with the same amount of pollutants, in which the tree cover was be used to determine which neighbourhood needs immediate attention over the other.

These four main factors were used to develop an algorithm to determine environmentally worst areas that require urgent attention within Toronto's neighbourhoods. This paper outlines this program algorithm and results from its use with Toronto data.

\section{Materials and Methods}

The primary tool chosen for data analysis was Python, a highlevel programming language. The data was from three files [1-3]. The name of each neighbourhood, neighbourhood ID, total area, total population, green rebate programs, green space, pollutant carcinogenic TEP score, non-carcinogenic TEP score, pollutants released to air, and tree cover were put to the first input file (Input. txt). The renewable energy information was put in another file which contained the following information: the name of each project, the neighbourhood it was built in, type, size, and year of initial installation.

If two neighbourhoods have the same amount of pollutants, then the neighbourhood with a smaller area will be considered to possess a worse environmental condition. This is under the assumption that the other factors are the same. Due to this, each factor is divided by the total geographical area of that neighbourhood. For instance, instead of using "pollutant carcinogenic TEP score", "pollutant carcinogenic TEP score per unit of area" is placed to make the evaluation fair.

The core algorithm is what follows:

1. Sort the neighbourhoods from the worst to the best according to each factor.

2. For each factor, separate the worst $25 \%$ to a new set.

3. Find the intersection of those four sets.

4. Process other information about each neighbourhood in the intersection and output more comprehensible information. 
For each factor, the worst 25 percent of neighbourhoods are separated. This " $25 \%$ " is an experimental value. According to the analysis of data in 2011 , when an index is not in the top $25 \%$, it becomes the average level, which is not the result desired. If the value is further lowered, however, some neighbourhoods with noticeably devastating environmental conditions can be ignored. Thus, "25\%" is a proper value to distinguish these neighbourhoods.

The intersection is used in step three for several reasons. First of all, all four factors chosen are critical values in the evaluation of the environmental condition. Together, the factors would decide whether a neighbourhood belongs to the worst group or not. Secondly, there can be many neighbourhoods which need environmen-

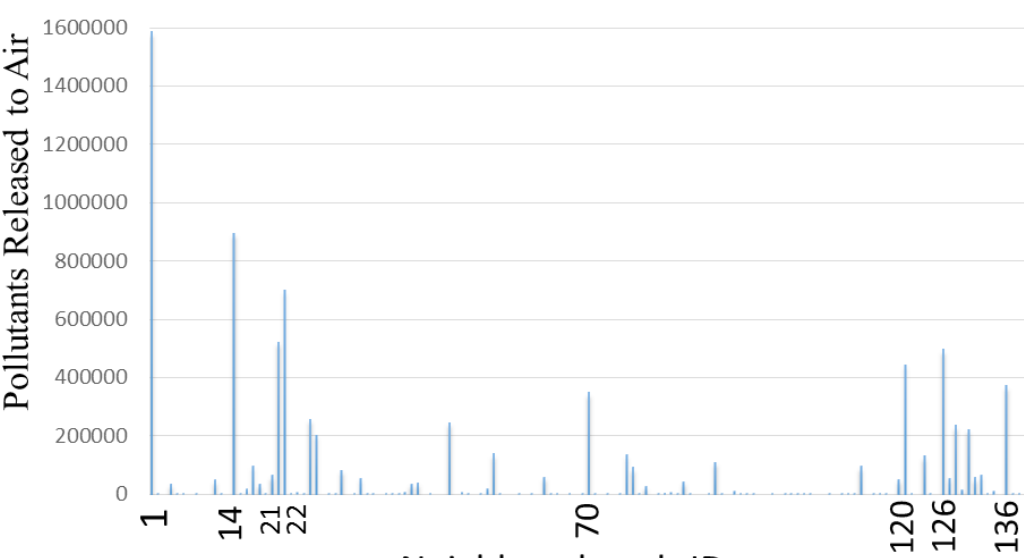

Neighbourhood ID

Figure 1: Pollutants Released to Air

Basically, the high-index group matches the final set, since few outliers exist. This implies that a neighbourhood with serious air pollution is more likely to have comparatively severe issues in all aspects.

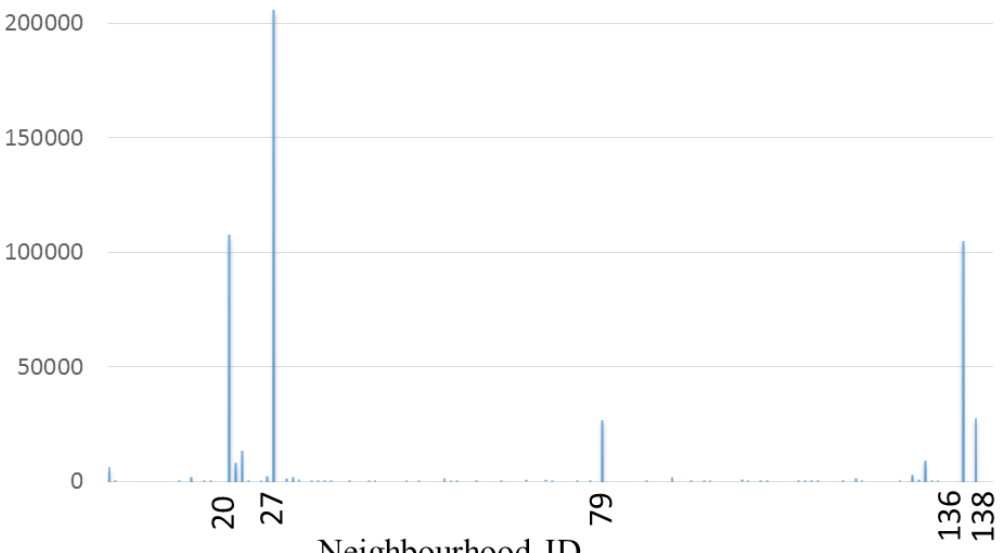

Figure 2: Pollutant Carcinogenic TEP Score.

Among the five Neighbourhoods with extraordinarily high indexes, only two make the final set. However, the other three should also be taken comprehensive consideration to be added to the group of urgency, for the Pollutant Carcinogenic TEP Score is always an unneglectable factor. tal improvement, but the output group expected is the focus - a small group of neighbourhoods in the most need for the overall improvement. Using the intersection, the program can concentrate on fewer neighbourhoods. Also, finding the intersection is easy and an efficient algorithmic step to manipulate.

The algorithm does several calculations in step four. After sorting, the associated rank is recorded to analyze the primary concern for each neighbourhood, with the notion that the top priority would be the highest-ranked factor.

Then the program analyzes the condition of the population density, green space, and green rebate programs. For the population density, "very low" represents bottom 25\%, "comparatively low" means bottom $25 \%$ to $50 \%$, "high" means top $50 \%$ to top $25 \%$, and "very high" means top $25 \%$. For green space, it is similar: "very little" for bottom 25\%, "comparatively little" for bottom $25 \%$ to $50 \%$, "comparatively satisfactory" for top $50 \%$ to $25 \%$, and "lots of" for top $25 \%$. For green rebate programs, the notions are "needs much more", "maybe add more", "many", and "a great number of". The program outputs the information about renewable energy plans. If the year the project was installed is before the year other data was collected, the program will remind the user to notice that.

\section{Results}

The program was run using Toronto data. Of the 12 neighbourhoods outputted, only 5 of them have renewable projects. Installing more renewable projects will be a way to improve the environment. Replacing industries that generate huge pollution and utilizing modern technology to generate greener and cleaner power will be the solution to the problem in the long run. In those neighbourhoods, renewable energy plans need more support.

Those neighbourhoods were expected to have few people, but many of those neighbourhoods have 10,000 to 20,000 , even over 30,000 people.

For most neighbourhoods, "the priority" determined by the program is either to reduce carcinogenic pollutants or to reduce pollutants released to air. Actually, the number of polluting facilities has a correlation coefficient of 0.864 with the amount of pollutants released to air. To further utilize the result, the companies producing the pollutants in those neighbourhoods need to be found. Setting up a tighter limit on the allowed numbers of pollutants released can be an effective method to reduce the values. 
From Open Data Toronto's dataset, only data from 2008 and 2011 were accessible, which limited the analysis to a certain level. The data from 2011 is the raw data, but the program can analyze any year's data as long as the input data are formatted properly. In this way, the user can use the program to determine next year's focus on environmental improvement plans.

\section{Discussion}

The algorithm only uses simple ranking and the intersection to find the answer set, so it is possible that there are some outliers during the analysis. The current ranking system cannot identify the gap between one value and another. This gap can be extremely large, which should also be considered. Using the intersection to find the answer set is not a perfect method, because a neighbourhood has to be top $25 \%$ in all four factors to make the final set. It causes the program to overlook some neighbourhoods which have very serious problems in only one or two fields. By visualizing the information, however, it is shown that although the algorithm is not perfect, the result still makes sense and meets the main objectives.

The figures present neighbourhoods and their index of pollutants. We can compare those neighbourhoods with the ones that make the final set to find the outliers.

Figure 1 shows pollutants released to the air data. Neighbourhoods with noticeably high index are 1, 14, 21, 22, 70, 120, 126, and 136. Neighbourhoods in the final set are 1, 14, 21, 70, and 126 .

Figure 2 shows pollutant carcinogenic TEP score data. Neighbourhoods with noticeably high indexes are 20, 27, 79, 136, and 138. Neighbourhoods in the final set are 27, and 79.

Figure 3 shows information about non-carcinogenic pollution. Although the non-carcinogenic pollution may not be as harmful to people, it still contributes to the unpleasant environmental conditions of neighbourhoods.

Figure 4 shows the tree cover of the neighbourhoods.

Outliers help determine methods of improvement to the

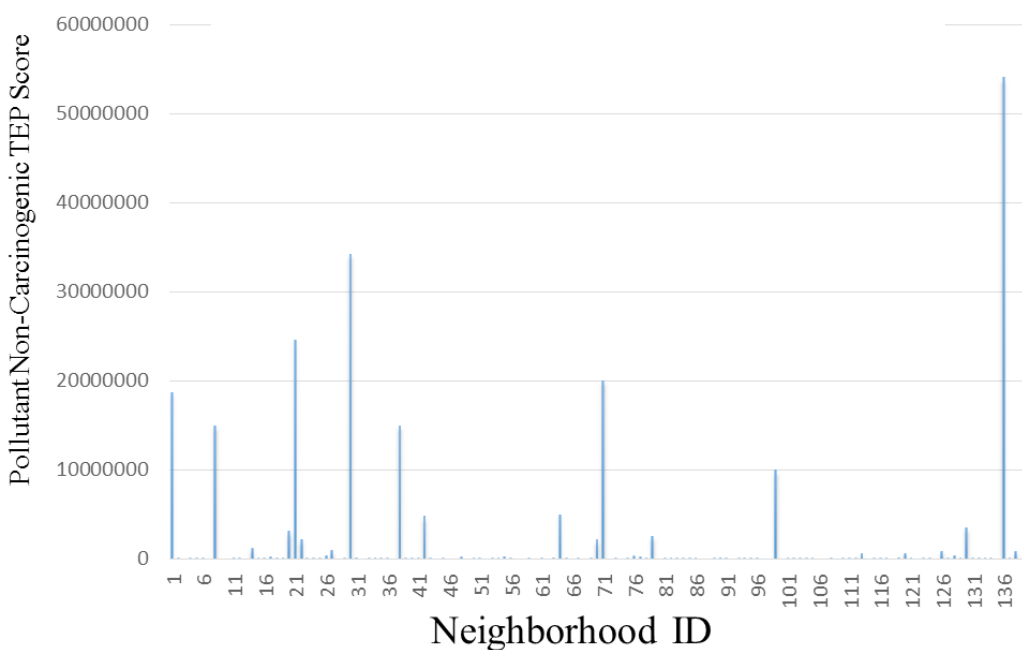

Figure 3: Pollutant Non-Carcinogenic TEP Score.

Non-carcinogenic pollutants, though not as important as those which are carcinogenic, can contribute to the build-up of greenhouse gases and a longterm environmental influence, indicating an overall environmental standard. The sifting of this factor may further narrow the scope of the final set.

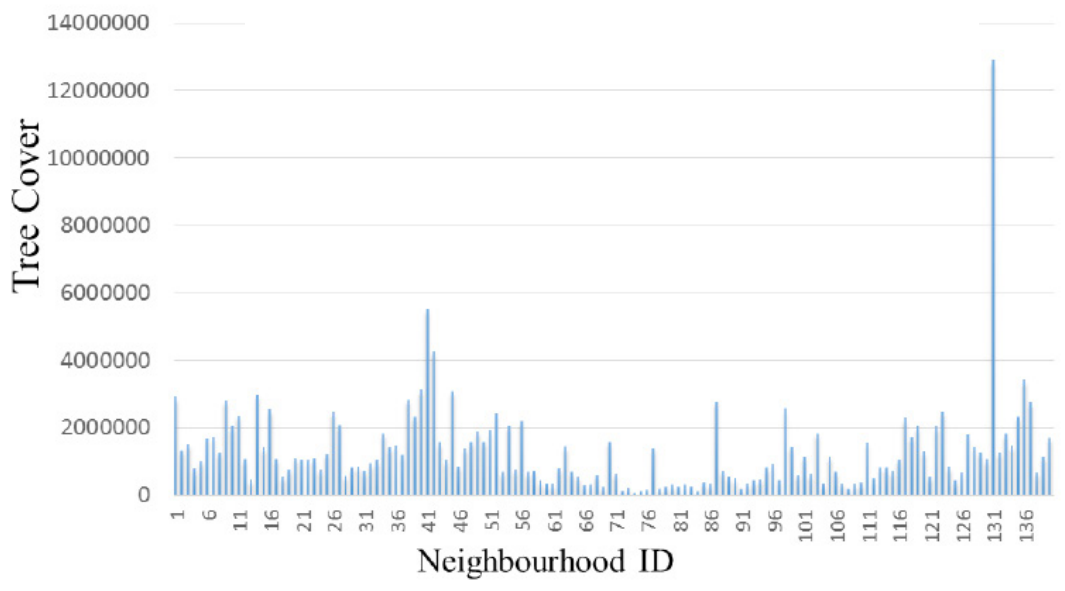

Figure 4: Tree Cover.

Planting trees still being an important means of environmental improvement, tree cover, as one of the four main factors chosen, works effectively to help choose a group of immediate attention. Thus, given that all other factors are similar, neighborhoods with less tree cover should be of priority. program. The previous method uses "25\%" as the experimental value to determine the "average level", but this can be solved using the variation of standard deviations. The ranking system of the redesigned algorithm has also been improved.

After sorting the data, find a group of data with small standard deviation while trying to keep that group as big as possible. This group is the "average" group.

The original group is the whole group. The group should be short- ened from both ends. (The data has been sorted)

Once the standard deviation varies less than $5 \%$ after being shortened, stop shortening on that side.

Thus, the "average group" is found and the group worse than the "average group" is the worst group.

Get the average value of each of the worst group. (There are four main factors, so there are four "worst groups".)

Any particular neighbourhood with a value $30 \%$ worse than the av- 
erage value calculated should be directly put into the answer set.

For other neighbourhoods, find the intersection of four sets.

To extend beyond this project, more research can be done on these neighbourhoods to find and identify specific problems for each neighbourhood.

\section{Conclusion}

While using an effective algorithm to analyze real, empirical data, the program designed suggests ways to improve communities' environments, which would better the quality of life in Toronto. The information was presented in a comprehensible way through our program. This study can help the environmental department of Toronto to determine a focus of work, and raise awareness in the neighbourhoods most in need of environmental improvement.

\section{Acknowledgements}

Thank you to Ms. Elissa Gelleny, for her support throughout this process. Ms. Gelleny is the mathematics and computer science teacher as well as the Moderator of our programming team. Her suggestions helped us move forward at many critical points.

Thank you to Dr. Danny Stout, for his consultations regarding our initial ideas and plans for this project.

Thanks to the entire team of involved staff from STEM Fellowship, SciNet, and SAS for the opportunity to compete with Big Data. Their efforts are greatly appreciated.

\section{Appendix}

Technical Methodology. The analysis program is called "analyze. py". It reads from two files: one is "Input.txt", which contains the information about the environment; the other one is "Input_2.txt", which contains the information about the renewable energy projects. The input files obey the correct format strictly in order for the program to run without error. The file "Input.txt", each line must contain the following information in order: the name of neighbourhood, ID, total area, population, green rebate programs, green space, pollutant carcinogenic TEP score, pollutant non-carcinogenic TEP score, pollutants released to air, and tree cover. A space may be retained between every piece of information. As for the file "Input_2.txt", the following information needs to be contained in each line in the proper order: the neighbourhood (where the project was installed) ID, the name of the project, the type of the project, the size the project (ekW), and the year when the project was installed successfully. Note that while inputting the information into this file (Input_2.txt), the separation symbol is "II" instead of a space. After the user finishes the input of the information, the user may close the files, and run the Python program "analyze.py" in the IDLE shell. A user input of the year of data collection will be prompted, and then the program will output the analysis result.

\section{References}

1. City of Toronto [Internet]. Wellbeing Toronto - Environment - Environment - Data catalogue. [Place unknown]: Social Development, Finance \& Administration; 2014, December 17 [cited 2015, December 20]. Available from: http://www1.toronto.ca/wps/portal/contentonly?vgnextoid=67f6 d05685a0c410VgnVCM10000071d60f89RCRD\&vgnextchannel=74a6e03bb8d1e310VgnVCM10000071d60f89RCRD.

2. City of Toronto [Internet]. Wellbeing Toronto - Locations and mapping - Demographics - Data catalogue. [Place unknown]: Social Development, Finance \& Administration; 2014, December 17 [cited 2015, December 20]. Available from: http://www1.toronto.ca/wps/portal/contentonly? vgnextoid=4482904ade9ea410VgnVCM10000071d60f89RCRD\&vgnextchannel=75d6e03bb8d1e310VgnVCM10000071d60f89RCRD.

3. City of Toronto [Internet]. Wellbeing Toronto - Environment - Renewable Energy Installations - Data catalogue. [Place unknown]: Facilities Management - Energy and Strategic Initiatives; 2015 January [cited 2015, December 20]. Available from: http://www1.toronto.ca/wps/portal/con tentonly?vgnextoid=1fd4736a37ff0310VgnVCM1000003dd60f89RCRD\&vgnextchannel=74a6e03bb8d1e310VgnVCM10000071d60f89RCRD. 


\section{REVIEW}

\section{by Lewis Wynne-Jones}

\section{ThinkData Works}

One of the problems facing the adoption of open data research into analytic solutions or data-driven journalism is that data are frequently released in fragmented sets, their critical metadata is difficult to track down (or missing altogether) and potentially relevant corollaries are left unpublished. To arrive at a conclusion using open data is, therefore, a procedure that depends as much on patience and ingenuity as it does on data science. The authors of "Environmental Analysis of Toronto Neighbourhoods" exhibited these skills, and more, while tackling an issue that is notoriously difficult to quantify.

By isolating four environmentally significant factors (pollutant release, carcinogenic and noncarcinogenic toxic equivalency potentials, and tree cover) Zhang, Baek, Bogopolskiy and Palombo developed a cohesive and clear methodology through which they were able to draw relevant and scientifically rigorous conclusions about the Toronto neighbourhoods that are most affected by industrial pollution. The inclusion of tree cover as a metric that, when high, logically implies fewer buildings (and therefore fewer industrial properties) is an effective and creative solution to a few of the variables that would otherwise have undermined the conclusions made in this paper.

Zhang et al displayed an analytical maturity by using raw data effectively and were thoughtful in their criticism of current environmental projects - eschewing bold strokes for even-tempered and clearly articulated calls to action. By identifying the neighbourhoods that are at risk, they called for a renewed environmental effort on the one hand and more accountability on the part of polluting entities on the other. At every point in their research they adhered to a cohesive and clear structure, making this paper not only well researched, but well written.

Although the breadth of this paper was undoubtedly limited to the data that were made available, it would have been interesting to see how industrial production per capita related to the study, or how congestion in high-traffic areas could be applied as a corollary. Similarly, climate data could have been overlaid on the results in order to refine and otherwise substantiate their data. Furthermore, access to more timely data (by using Wellbeing Toronto as a resource Zhang et al were limited to data from 2011 - the source has since updated to include 2014 data) would have allowed the researchers to better test their conclusions.

Increasingly, it is necessary for government to make decisions based not on a perceived need for environmental regulation, but rather on data-driven conclusions that take the guesswork out of environmental policy. The authors of "Environmental Analysis of Toronto Neighbourhoods" were able to articulate a difficult and pressing concern, and it is clear that - provided they have access to useful data - they possess the digital skills to achieve even greater insight in the future. 